\title{
TENS augments blood flow in somatotopically linked spinal cord segments and mitigates compressive ischemia
}

\author{
BS Budgell, G Sovak and D Soave
}

\begin{abstract}
Study Design: This was an acute basic physiological study in anesthetized adult male rats.
Objectives: The purpose of this study was to determine, in an animal model, whether innocuous somatic stimulation, in the form of transcutaneous electrical nerve stimulation (TENS), could produce a sustained augmentation of spinal cord blood flow, and whether this effect was robust in the face of relatively mild, non-destructive compression of the spinal cord.
\end{abstract}

Setting: Neurophysiology laboratory, Canadian Memorial Chiropractic College, Toronto, Canada.

Methods: In anesthetized adult male Wistar rats, spinal cord blood flow was measured with laser Doppler flowmetry during 5- and 15-min epochs of TENS stimulation in uncompressed and compressed lumbar spinal cord.

Results: TENS applied to the L4/L5 dermatomes was associated with augmentation of blood flow in somatotopically linked spinal cord segments. This augmentation was robust in the face of non-destructive compression of the spinal cord, was sustained for periods of stimulation up to $15 \mathrm{~min}$ and occurred in the absence of any change in the mean arterial blood pressure.

Conclusions: TENS augments spinal cord blood flow in the uncompressed spinal cord and during acute, non-destructive spinal cord compression. It remains to be seen whether similar results can be achieved in chronically compressed spinal cord and spinal nerve roots, and whether these results have clinical implications in human syndromes of spinal cord compression.

Sponsorship: This work was supported by internal research funds from Canadian Memorial Chiropractic College.

Spinal Cord (2014) 52, 744-748; doi:10.1038/sc.2014.120; published online 22 July 2014

\section{INTRODUCTION}

The functional integrity of the spinal cord and spinal nerve roots is, of course, dependent upon adequate perfusion and the availability of oxygen. As one might expect, laboratory investigations of spinal cord/ nerve root function often model the most destructive lesions in which normal anatomical and physiological relationships have been disrupted. ${ }^{1,2}$ In rat models of traumatic spinal cord injury, it has been demonstrated that both the extent and nature of neuronal damage are linked to the degree and duration of ischemia. ${ }^{3,4}$ Compressive injury has been shown to cause an immediate and sustained decrease in local spinal cord blood flow (SCBF) in rat models. ${ }^{3,5,6}$ Furthermore, augmentation of SCBF is associated with improved recovery of motor function in rat models of spinal cord compression. ${ }^{7,8}$ Less is known about spinal cord responses to milder compression.

With regard to the therapeutic potential of physical therapies, it is understood that various forms of somatic stimulation increase SCBF in animal models. Recent studies in urethane-anesthetized adult male rats confirm that innocuous somatic stimulation (cutaneous brushing) results in a segmentally organized reflex augmentation of regional SCBF in the intact spinal cord. ${ }^{9}$ Furthermore, these studies suggest that, whereas neuronal activation is probably the most important mechanism underlying increased SCBF, alpha-adrenergic receptors also participate in this process. ${ }^{10,11}$ The studies cited ${ }^{9-11}$ employed stimulation periods of 30-60 s, and therefore it is unclear whether animals might quickly habituate to stimulation or whether the increases in SCBF could be maintained for periods likely to have clinical relevance in humans. Furthermore, these studies were conducted in intact, non-compressed spinal cords and, therefore, to date, there are no published reports of somatic stimulation mitigating the effects of compression on spinal cord or nerve root blood flow.

Therefore, the present study was designed to determine whether innocuous somatic stimulation, in the form of transcutaneous electrical nerve stimulation (TENS), could produce a sustained augmentation of SCBF, and whether this effect was robust in the face of relatively mild, non-destructive compression of the spinal cord.

\section{MATERIALS AND METHODS}

\section{General surgical procedures}

Twelve-week-old male Wistar rats $(n=20$; mean weight $347 \pm 14 \mathrm{~g})$ were anesthetized with urethane $\left(1.3 \mathrm{~g} \mathrm{~kg}^{-1}\right.$ intraperitoneally (i.p.)). Core temperature was monitored (TCAT-2LV Temperature Controller; David Kopf) via a rectal probe and maintained at $37.0 \pm 0.5^{\circ} \mathrm{C}$. A carotid artery and jugular vein were catheterized to monitor blood pressure, and for the provision of supplementary anesthesia, respectively. Animals were allowed to breathe spontaneously. Data were acquired via a PowerLab $8 / 30$ data acquisition system (ADInstruments, Boulder, CO, USA).

Disposable EKG electrodes (Blue Sensor, Ambu A/S, Denmark) were placed on the skin over the dorsum of the right hind paw and over the right sacroiliac joint-approximately the L4-L5 dermatomes ${ }^{12}$ and attached to a stimulus isolator (ML180, ADInstruments), which delivered TENS-like stimulation, that is, $0.5 \mathrm{~ms}^{2}$ waves at $100 \mathrm{~Hz}$ with intensities set at $2.0,3.0$ or $3.5 \mathrm{~mA}$. These intensities were selected based on the physiological responses seen in pilot experiments wherein $2.0 \mathrm{~mA}$ caused no visible muscle contraction but 
occasional and intermittent palpable fasciculation in limb muscles, $3.0 \mathrm{~mA}$ routinely caused fasciculations and occasionally caused muscle twitching, and $3.5 \mathrm{~mA}$ routinely caused muscle twitching and occasionally caused sustained tetany of large hind-limb muscles.

The L4-L6 lumbar spinal cord was exposed by T13/L1 laminectomy, with the dura mater left intact. The T12 and L2 spinous processes were stabilized with vertebral clamps. A laser Doppler probe (OxyFlo Probe, Oxford Optronix, UK) mounted on a pressure transducer (Fort100, World Precision Instruments, Sarasota, FL, USA) was lowered, using a micromanipulator (UM-IPF, Narishige, East Meadow, NY, USA), onto the surface of the dura mater that was depressed until it just touched the dorsal surface of the spinal cord (Figure 1). Positioning of the probe targeted the L4-L5 level of the spinal cord. Spinal cord levels were confirmed at the end of each experiment by tracing spinal nerve rootlets to the intervertebral foramina through which they appeared to exit. The laser Doppler flow signal represents the number and velocity of red blood cells moving through the tissue to a depth of $\sim 1-2 \mathrm{~mm}$ beneath the probe tip. The laser Doppler flow signal therefore reflects relative blood flow and is expressed in millivolts but has been found to correlate very well with changes in absolute tissue blood flow measured by other means. ${ }^{13}$ The advantage of laser Doppler flow is that it is non-invasive and reflects real-time changes in blood flow.

\section{Experimental series 1}

In each of six animals, in order to determine effect sizes and optimum stimulation parameters, 5-min epochs of stimulation were applied three times at each intensity $(2.0,3.0$ and $3.5 \mathrm{~mA})$, with $\sim 2$ - to 3-min interstimulus intervals. SCBF and force applied through the laser Doppler probe were monitored before the onset of stimulation, for the duration of stimulation, and following stimulation until SCBF had stabilized at prestimulation levels.

In four of the six animals, the laser Doppler probe (tip diameter: $0.52 \mathrm{~mm}$ ) was subsequently lowered onto the spinal cord with sufficient force to reduce baseline SCBF by a target of 10-20\%. Hence, compression targeted a range of reduction in blood flow, not a specific force. SCBF does fluctuate spontaneously, and spinal cord tissue undergoes a degree of plastic deformation under pressure. Thus, there was some degree of variability in the actual attenuation of SCBF. Nonetheless, the target level of SCBF attenuation was practical and did not produce gross tissue disruption (subsequently confirmed with histological examination). Five-minute epochs of stimulation were repeated in the compressed spinal cord at the three levels of current intensity.

\section{Experimental series 2}

Effect sizes from experimental series 1 (mean relative SCBF increase/s.d.; Table 1) provided minimum sample size estimates of six animals per stimulation intensity. Therefore, 14 animals were subjected to $3 \times 15$-min epochs of stimulation at a single current intensity, either $2.0 \mathrm{~mA}(n=7)$ or $3.0 \mathrm{~mA}(n=7)$, before and after compression of the spinal cord. Because of the relatively long periods of stimulation $(3 \times 15 \mathrm{~min}$, plus interstimulus intervals), and hence length of the entire experiment, multiple magnitudes of stimulation were not tested in each animal.

At the conclusion of SCBF measurements, sufficient anesthesia was given to ensure that the animals were deeply anesthetized. For histological examination of the spinal cord ( $n=5$ animals), the chest cavity was opened and the animals were perfused transcardially with cold saline followed by $10 \%$ neutral buffered formalin. The spinal cord from the T11 level to the caudal extreme was removed and transferred to $10 \%$ neutral buffered formalin for $48 \mathrm{~h}$. Subsequently, the tissues were subjected to routine histological processing with hematoxylin and eosine and Luxol fast blue staining. Serial sections $(6 \mu \mathrm{m})$ were evaluated qualitatively to determine whether the experimental paradigm was associated with any histomorphological changes.

\section{Statistical analysis}

For both series of experiments, SCBF data from the three trials of stimulation at each intensity were averaged. At each stimulus intensity this averaged SCBF (i) within 1 min of the onset of stimulation, a period that normally showed a transient peak in SCBF, (ii) over the entire period of stimulation (series 1: $5 \mathrm{~min}$; series 2: $15 \mathrm{~min}$ ) and (iii) in the final minute of stimulation were calculated to characterize blood flow changes throughout the course of

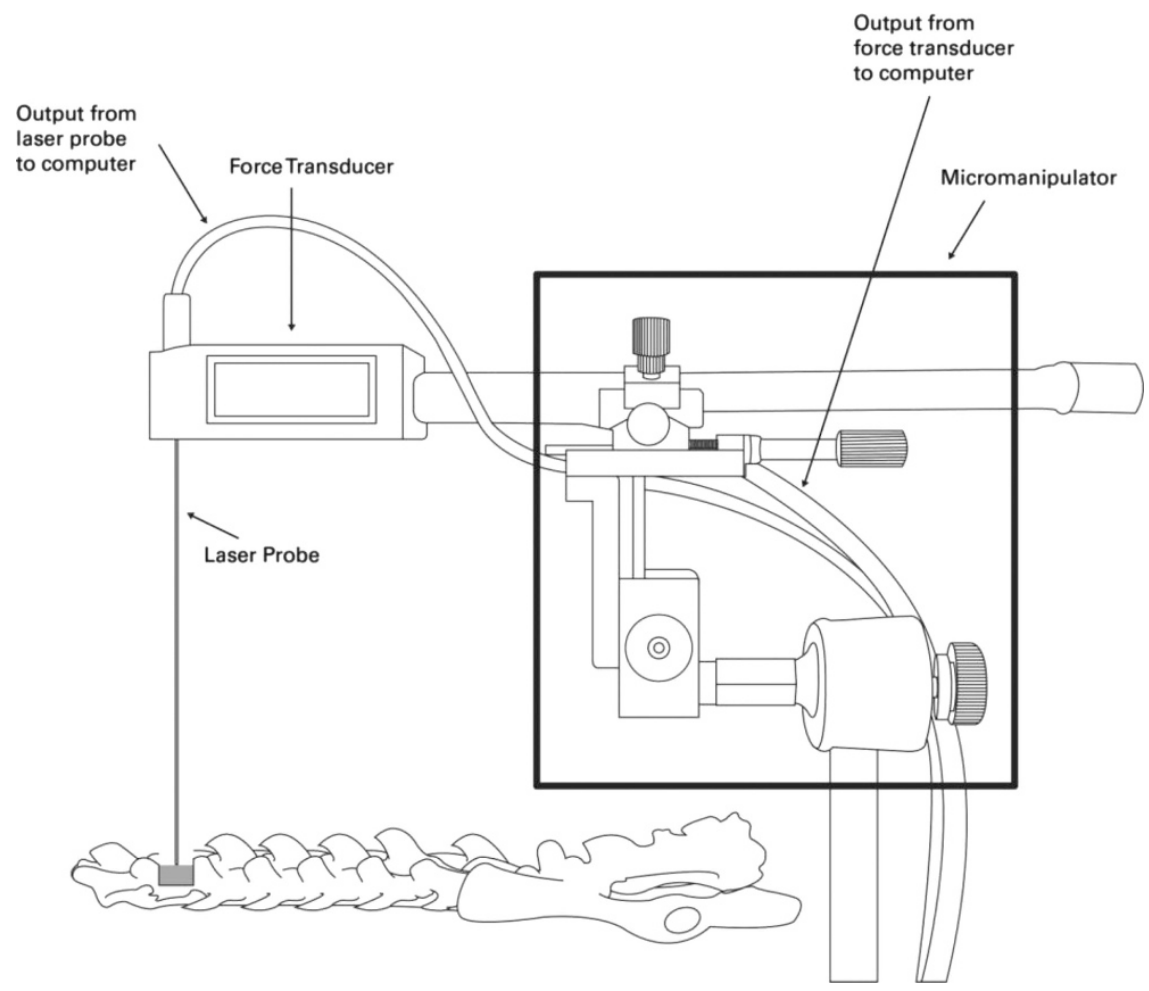

Figure 1 Simultaneous measurement of SCBF and compression of the spinal cord. A laser Doppler probe is fixed to a force transducer that, in turn, is mounted on a micromanipulator allowing regulation of force applied by the probe head to the spinal cord with simultaneous measurement of SCBF and pressure applied to the spinal cord. In this figure, the probe is shown contacting the spinal cord exposed by an L1 laminectomy. 
stimulation, and between stimulus intensities to determine whether stimulus intensity affected the magnitude of SCBF. Summary statistics for experimental series 1 were calculated for sample size determination in experimental series 2 . Relative SCBF change was analyzed in experimental series 2 using one-sample $t$-tests at each epoch and stimulation intensity. Non-parametric Wilcoxonsigned rank tests were also performed for comparison as parametric statistical assumptions may not be confirmed with small sample sizes $(n=7)$.

\section{Statement of Ethics}

We certify that all applicable institutional and governmental regulations concerning the ethical use of animals were followed during the course of this research.

\section{RESULTS}

Before compression of the spinal cord, the force exerted via the laser Doppler probe tip on the intact dura mater across all trials was $1.66 \pm 0.36 \mathrm{~g}$ (mean \pm s.d.) equivalent to $600 \mathrm{~mm} \mathrm{Hg}$. Following the application of compression, the force exerted via the laser Doppler probe tip was $3.38 \pm 1.33 \mathrm{~g}$ (mean \pm s.d.) equivalent to $1220 \mathrm{~mm} \mathrm{Hg}$.
The actual pressure exerted on the spinal cord itself could not be measured. However, compression resulted in an average attenuation of SCBF of $19 \pm 12 \%$ (mean \pm s.d.).

\section{Experimental series 1}

TENS applied to the hind limb for periods of up to $5 \mathrm{~min}$ produced a substantial augmentation of SCBF in the uncompressed and compressed spinal cord (Figures $2 \mathrm{a}$ and $\mathrm{b}$, Table 1). Whereas Kurosawa and colleagues $^{9-11}$ had reported unimodal responses in SCBF with 30 and $60 \mathrm{~s}$ epochs of cutaneous stimulation, in the current study there was an initial and rapid rise in SCBF within the first minute followed by a decline to a sustained plateau that scarcely seemed to decay throughout the remaining period of stimulation. The magnitude of SCBF enhancement was influenced by the magnitude of the applied current (Table 1). In all phases of stimulation-peak, tail and over the entire 5min duration-average augmentation appeared to be greater with 3.0 and $3.5 \mathrm{~mA}$ than with $2.0 \mathrm{~mA}$, and occurred without any significant change in the mean arterial blood pressure (two-tailed paired $t$-test,

Table 1 Augmentation of SCBF, mean $+(95 \%$ Cls $)+($ Cohen's d), during TENS at three stimulation intensities and in comparison with normalized pre-stimulation SCBF

\begin{tabular}{|c|c|c|c|}
\hline Pre/post compression & Peak mean (95\% Cl) and (effect size) & Tail mean $(95 \% \mathrm{Cl})$ and (effect size) & Total ( $5 \mathrm{~min})$ mean $(95 \% \mathrm{Cl}$ ) and (effect size) \\
\hline \multicolumn{4}{|l|}{$2 m A$} \\
\hline Pre $(n=6)$ & $1.26(1.14,1.38)(2.3)$ & $1.17(1.08,1.27)(1.8)$ & $1.21(1.10,1.31)(2.2)$ \\
\hline Post $(n=4)$ & $1.08(1.04,1.11)(4.2)$ & $1.10(1.01,1.20)(1.6)$ & $1.09(1.03,1.15)(2.4)$ \\
\hline \multicolumn{4}{|l|}{$3 m A$} \\
\hline Pre $(n=6)$ & $1.42(1.25,1.58)(2.8)$ & $1.33(1.17,1.50)(2.0)$ & $1.37(1.20,1.55)(2.2)$ \\
\hline Post $(n=4)$ & $1.14(1.05,1.24)(2.2)$ & $1.11(1.06,1.15)(4.4)$ & $1.11(1.05,1.17)(2.9)$ \\
\hline \multicolumn{4}{|l|}{$3.5 \mathrm{~mA}$} \\
\hline Pre $(n=6)$ & $1.45(1.25,1.65)(2.4)$ & $1.33(1.18,1.49)(2.2)$ & $1.39(1.20,1.57)(2.3)$ \\
\hline Post $(n=4)$ & $1.18(1.05,1.31)(2.2)$ & $1.13(1.06,1.19)(3.4)$ & $1.14(1.06,1.22)(2.8)$ \\
\hline
\end{tabular}

Abbreviations: $\mathrm{Cl}$, confidence interval; SCBF, spinal cord blood flow; TENS, transcutaneous electrical nerve stimulation.

Augmentation is noted for both uncompressed ('Pre') and compressed ('Post') spinal cord.

a

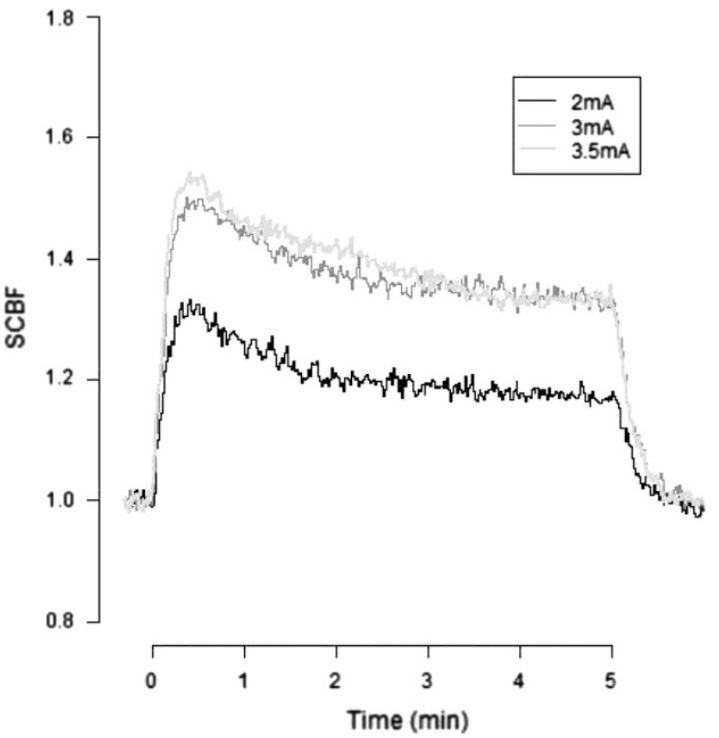

b

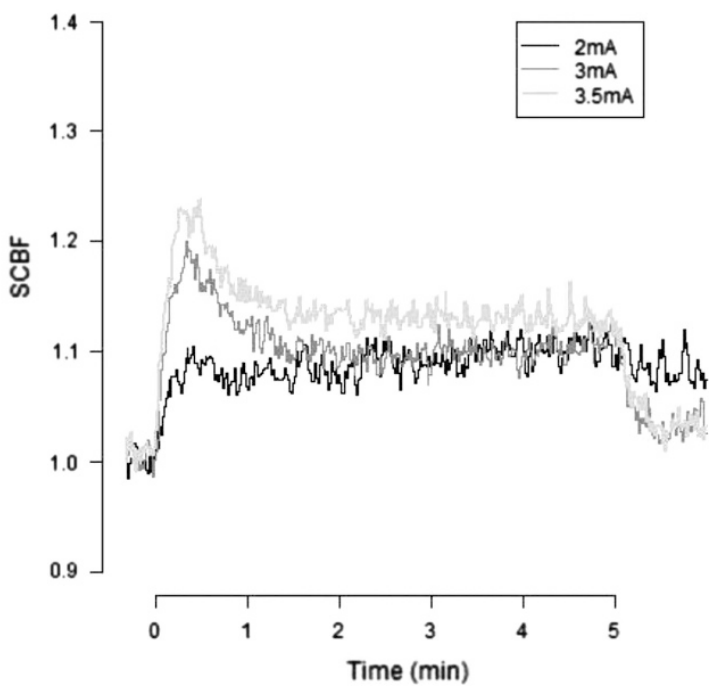

Figure 2 SCBF during $5 \mathrm{~min}$ of TENS stimulation. SCBF measured for $20 \mathrm{~s}$ before and then during $5 \mathrm{~min}$ of TENS stimulation (Time $=0-=5 \mathrm{~min}$ ) at three current intensities before spinal cord compression (a) in six animals, and during spinal cord compression (b) in four animals. 
$P>0.05$ in all cases). In the uncompressed cord, the augmentation of SCBF achieved with $3.0 \mathrm{~mA}$ stimulation was equivalent to that obtained with $3.5 \mathrm{~mA}$, even though the stronger stimulation usually elicited a pronounced and sustained contraction of hind limb muscles.

\section{Experimental series 2}

TENS stimulation of 15 -min duration at either 2.0 or $3.0 \mathrm{~mA}$ produced augmentation of SCBF for the entire duration of stimulation in both uncompressed and compressed spinal cord (Figures 3a and b, Table 2), without any significant change in the mean arterial blood pressure (two-tailed paired $t$-test, $P>0.05$ in both cases).

In the uncompressed spinal cord and at both intensities of current, the initial peak blood flow, the average blood flow over the $15 \mathrm{~min}$ of stimulation and the sustained blood flow in the final minute of stimulation-the 'tail'-were significantly higher than at baseline before the onset of stimulation (Table 2). Following compression of the spinal cord, both intensities of TENS (2.0 and $3.0 \mathrm{~mA}$ ) caused augmentation of SCBF. However, only in the first minute of stimulation-the 'peak' - did the mean augmentation with $3.0 \mathrm{~mA}$ appear to exceed the augmentation of SCBF achieved with $2.0 \mathrm{~mA}$.
The magnitudes of compression used were sufficient to impair blood flow without causing macroscopically obvious damage. Representative histological sections from the area of compressed spinal cord in five animals confirmed that compression did not produce any distortion of spinal cord tissues, disruption of normal anatomical boundaries or bleeding into the spinal cord.

\section{DISCUSSION}

TENS stimulation applied across the L4 and L5 dermatomes in the anesthetized rat causes an immediate increase in blood flow in the associated segments of the lumbar spinal cord. Augmentation of SCBF is evident with stimulation parameters that are likely to be innocuous in conscious animals, although the degree of augmentation is linked to stimulus intensity in a non-linear manner. Specifically, stimulation sufficient to induce sustained muscle contraction in the hind limb is not associated with greater augmentation of SCBF than stimulation of lower intensity that only induces intermittent contraction or fasciculation. Augmentation of SCBF was sustained for periods of stimulation up to $15 \mathrm{~min}$, a period that is used to assess mobility in patients with lumbar spinal stenosis. ${ }^{14}$ Cessation of TENS was
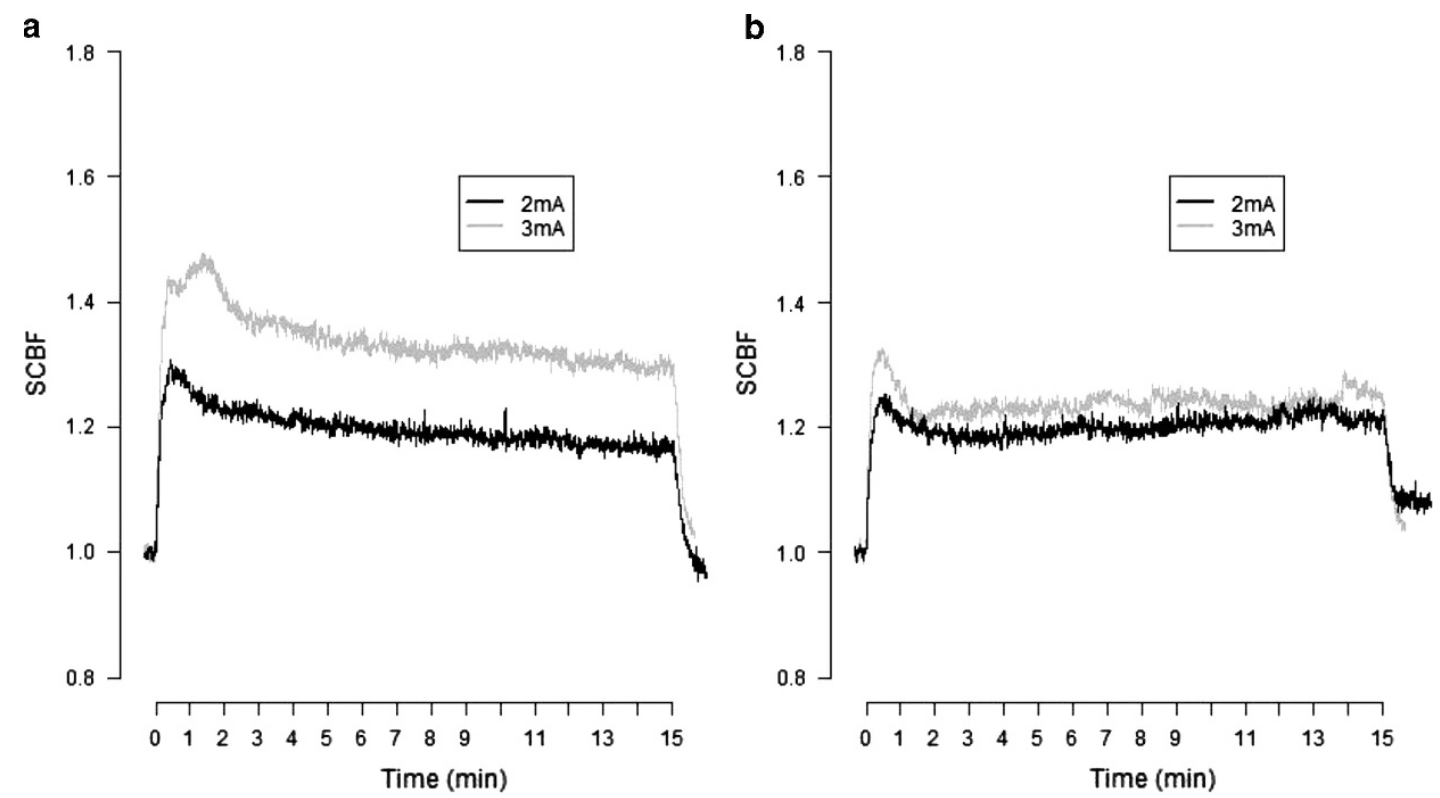

Figure 3 SCBF during $15 \mathrm{~min}$ of TENS stimulation. SCBF measured for $20 \mathrm{~s}$ before and then during $15 \mathrm{~min}$ of TENS stimulation (Time $=0-15 \mathrm{~min}$ ) at two current intensities, each in seven animals, before spinal cord compression (a), and during spinal cord compression (b).

Table 2 Augmentation of SCBF $+(95 \%$ confidence intervals $)+(P$-value $)$ during TENS at two stimulation intensities and in comparison with normalized prestimulation SCBF

\begin{tabular}{|c|c|c|c|}
\hline Pre/post compression & Peak mean $(95 \% \mathrm{Cl})$ (t-test $(\mathrm{P}) /$ Wilcoxon $(\mathrm{P}))$ & Tail mean (95\% Cl) (t-test (P)/Wilcoxon (P)) & Total mean $(95 \% \mathrm{Cl})$ (t-test (P)/Wilcoxon (P)) \\
\hline \multicolumn{4}{|l|}{$2 m A$} \\
\hline Pre $(n=7)$ & $1.25(1.15,1.35)(0.001 / 0.016)$ & $1.17(1.08,1.26)(0.004 / 0.016)$ & $1.20(1.11,1.29)(0.002 / 0.016)$ \\
\hline Post $(n=6)$ & $1.21(1.09,1.32)(0.006 / 0.031)$ & $1.21(1.08,1.34)(0.008 / 0.031)$ & $1.20(1.09,1.32)(0.007 / 0.031)$ \\
\hline \multicolumn{4}{|l|}{$3 m A$} \\
\hline Pre $(n=7)$ & $1.38(1.24,1.52)(0.0006 / 0.16)$ & $1.29(1.12,1.47)(0.007 / 0.016)$ & $1.34(1.16,1.52)(0.003 / 0.016)$ \\
\hline Post $(n=7)$ & $1.27(1.15,1.39)(0.001 / 0.016)$ & $1.26(1.18,1.34)(0.0002 / 0.016)$ & $1.24(1.16,1.32)(0.0004 / 0.016)$ \\
\hline
\end{tabular}

Abbreviations: $\mathrm{Cl}$, confidence interval; SCBF, spinal cord blood flow; TENS, transcutaneous electrical nerve stimulation.

Augmentation is noted for both uncompressed ('Pre') and compressed ('Post') spinal cord. P-values were obtained using both parametric one-sample $t$-tests and non-parametric Wilcoxon-signed rank tests as parametric statistical assumptions may not be confirmed with small sample sizes. 
associated with an immediate decline in blood flow that returned to pre-stimulation levels in about $1 \mathrm{~min}$. The augmentation of SCBF by TENS stimulation occurred in the absence of any change in the mean arterial blood pressure. Punctate compression of the spinal cord with pressures insufficient to produce macroscopic or histological evidence of tissue disruption led to, on average, an $\sim 19 \%$ attenuation of SCBF. However, this attenuation was mitigated by TENS stimulation.

The degrees of augmentation of SCBF reported herein are quite similar to those previously reported with innocuous mechanical stimulation (stroking) of the skin. ${ }^{9,10}$ Augmentation of cervical SCBF has also previously been reported in the cat in response to direct electrical stimulation of isolated cutaneous branches of the radial nerve. ${ }^{15}$ In the present study, with intensities of 2.0 and $3.0 \mathrm{~mA}$, augmentation of SCBF was sustained for 15-min epochs of stimulation in both uncompressed and compressed spinal cord.

As the laser Doppler beam is rapidly attenuated as it passes through tissues, and as perfusion of the grey matter is substantially greater than that of the white matter, it is likely that such effects on SCBF as were recorded in this study were principally attributable to changes in perfusion of the dorsal grey matter. Furthermore, that augmentation of SCBF occurred in the absence of any change in the mean arterial blood pressure suggests that neuronal activation, rather than a systemic cardiovascular response to stimulation, was an important driver of enhanced spinal cord perfusion.

Impact and clip compression models of spinal cord injury often result in immediate macroscopic evidence of vascular damage. ${ }^{16}$ Depending upon the parameters of injury, there may be gross intraparenchymal bleeding or edema, as well as loss of microcirculation leading to longer-term pathological processes. ${ }^{17,18}$ In contrast, histological examination of representative sections of spinal cord tissue taken from the compressed and control segments in this study showed no evidence of tissue disruption, for example, no disruption of normal tissue boundaries and no bleeding. However, no analysis was undertaken to rule out that biochemical changes and alternative histological techniques may well have revealed more subtle changes (for example, see Kobayashi et al. ${ }^{19}$ ). Such compression as did take place produced, on average, a 19\% decrease in SCBF. Depending upon the time point and intensity of stimulation, in compressed spinal cord TENS produced average augmentations of SCBF ranging from 8 to $27 \%$ (Tables 1 and 2). In other words, there was substantial mitigation of the ischemic effects of non-destructive compression. To place this effect into perspective, one rat model of lumbar stenosis reported a $30-50 \%$ reduction in SCBF associated with clear cut behavioural changes suggestive of intermittent claudication. ${ }^{20}$

\section{Limitations}

The current experiments did not model a chronic compressive syndrome. Rather, they examined short-term physiological responses to an acute, punctate compression of the spinal cord. Neural tissues subjected to slowly evolving compression may well undergo adaptive changes that would alter responses to somatic stimulation. The current studies were conducted in rats that are much more resilient in the face of traumatic spinal cord injury than humans. Furthermore, the majority of clinical cases of lumbar stenosis involve the lower lumbar spine and hence impingement of the spinal nerve roots. The effect of TENS on perfusion of these tissues was not investigated in this study. Hence, caution should be exercised in extrapolating these results to clinical effects in humans.

\section{CONCLUSIONS}

TENS stimulation applied to lumbar dermatomes in the anesthetized rat augments SCBF in somatotopically linked spinal segments, and mitigates the ischemic effect of acute, mild, non-destructive compression of the spinal cord.

\section{DATA ARCHIVING}

There were no data to deposit.

\section{CONFLICT OF INTEREST}

The authors declare no conflict of interest.

\section{ACKNOWLEDGEMENTS}

This work was supported by internal research funds from Canadian Memorial Chiropractic College.

1 Agrawal SK, Fehlings MG. Mechanisms of secondary injury to spinal axons in vitro: Role of $\mathrm{Na}^{+}, \mathrm{Na}^{+}-\mathrm{K}^{+}-$ATPase, the $\mathrm{Na}^{+}-\mathrm{H}$ exchanger, and the $\mathrm{Na}^{+}-\mathrm{Ca}^{2+}$ exchanger J Neurosci 1996; 16: 545-552.

2 Agrawal SK, Fehlings MG. Role of NMDA and Non-MNDA ionotropic glutamate receptors in traumatic spinal cord axonal injury. J Neurosci 1997; 17: 1055-1063.

3 Fehlings M, Tator C, Linden R. The relationships among the severity of spinal cord injury, motor and somatosensory evoked potentials and spinal cord blood flow. Electroencephalogr Clin Neurophysiol 1989; 74: 241-259.

4 Lu K, Liang C, Chen H, Chen S, Hsu H, Liliang P et al. Injury severity and cell death mechanisms: effects of concomitant hypovolemic hypotension on spinal cord ischemiareperfusion in rats. Exp Neurol 2004; 185: 120-132.

5 Ross I, Tator C. Further studies of nimodipine in experimental spinal cord injury in the rat. J Neurotrauma 1991; 8: 229-238.

6 Tei R, Kaido T, Nakase H, Sakaki T. Secondary spinal cord hypoperfusion of circumscribed areas after injury in rats. Neurol Res 2005; 27: 403-408.

7 Nakauchi K, Ikata T, Katoh S, Hamada Y, Tsuchiya K, Fukuzawa K. Effects of lecithinized superoxide dismutase on rat spinal cord injury. J Neurotrauma 1996; 13: 573-582.

8 Nakai I, Takenobu Y, Takimizu H, Akimaru S, Maegawa H, Ito $\mathrm{H}$ et al. Effects of OP1206 alpha-CD on walking dysfunction in the rat neuropathic intermittent claudication model: comparison with mifedipine, ticlopidine and cilostazol. Prostaglandins Other Lipid Mediat 2003; 71: 253-263.

9 Kurosawa M, Watanabe O, Maruyama H, Budgell B. Responses of dorsal spinal cord blood flow to innocuous cutaneous stimulation in anesthetized rats. Auton Neurosci 2006; 126-127: 185-192.

10 Kurosawa M, Toda H, Watanabe A, Budgell B. Contribution of supraspinal and spinal structures to the responses of dorsal spinal cord blood flow to innocuous cutaneous brushing in rats. Auton Neurosci 2007; 136: 96-99.

11 Toda H, Maruyama H, Budgell B, Kurosawa M. Responses of dorsal spinal cord blood flow to noxious mechanical stimulation of the skin in anesthetized rats. J Physiol Sci 2008; 58: 263-270.

12 Takahashi Y, Nakajima Y, Sakamoto T. Dermatome mapping in the rat ahindlimb by electrical stimulation of the spinal nerves. Neurosci Lett 1994; 168: 85-88.

13 Phillips JP, Cibert-Goton V, Langford RM, Shortland PJ. Perfusion assessment in rat spinal cord tissue using photoplethysmography and laser Doppler flux measurements. J Biomed Opt 2013; 18: 3

14 Tomkins-Lane CC, Holz SC, Yamakawa KS, Phalke VV, Quint DJ, Miner J et al. Predictors of walking performance and walking capacity in people with lumbar spinal stenosis, low back pain and asymptomatic controls. Arch Phys Med Rehabil 2012; 93: 647-653.

15 Seki M, Maeda M. Effects of electrical stimulation of motor and cutaneous nerves on spinal cord blood flow. Spine 1993; 18: 1798-1802.

16 Young W. MASCIS spinal cord contusion model. In: Chen J, Xu ZC, Xu XM and Zhang JH (eds) Animal Models of Acute Neurological Injuries. Humana Press: Totowa, NJ, USA, 2009, pp 411-421.

17 Park YK, Tator $\mathrm{CH}$. Failure of topical DMSO to improve blood flow or evoked potentials in rat spinal cord injury. J Korean Med Sci 1998; 13: 638-644.

18 Figley SA, Khosravi R, Legasto JM, Tseng YF, Fehlings MG. Characterization of vascular disruption and blood-spinal cord barrier permeability following traumatic spinal cord injury. J Neurotrauma 2014; 31: 541-552.

19 Kobayashi S, Yoshizawa H, Shimada S, Guerrero AR, Miyachi M. Changes of blood flow, oxygen tension, action potential and vascular permeability induced by arterial ischemia or venous congestion on the spinal cord in canine model. J Orthop Res 2013; 31: 139-146.

20 Takenobu Y, Katsube N, Marsala M, Kondo K. Model of neuropathic intermittent claudication in the rat: methodology and application. J Neurosci Methods 2001; 104: 191-198. 